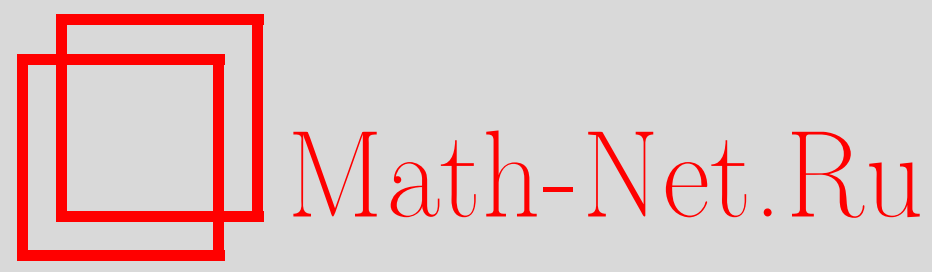

А. М. Стоколос, К одной проблеме А. Зигмунда, Матем. заметки, 1998, том 64, выпуск 5, 749-762

DOI: https://doi.org/10.4213/mzm1451

Использование Общероссийского математического портала Math-Net.Ru подразумевает, что вы прочитали и согласны с пользовательским соглашением http://www . mathnet.ru/rus/agreement

Параметры загрузки:

IP : 54.84 .234 .179

26 апреля 2023 г., 11:30:55

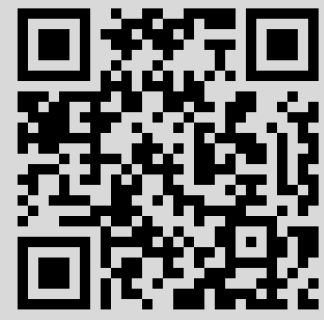




\section{К ОДНОЙ ПРОБЛЕМЕ А. ЗИГМУНДА}

\section{А. М. Стоколос}

Доказано, что существует суммируемая на $[0,1]^{2}$ функция, интеграл от которой недифференцируем в каждом из направлений, составляющих всюду плотное в $[0,2 \pi]$ множество, но дифференцируем сильным образом.

Библиограффия: 8 названий.

Классическая теорема Йессена-Марцинкевича-Зигмунда [1] утверждает сильную дифференцируемость интегралов функций из классов $L\left(\log ^{+} L\right)^{n-1}\left(I^{n}\right)$. С. Сакс [2] показал, что в любом классе $\Phi(L)\left(I^{n}\right)$, более широком чем $L\left(\log ^{+} L\right)^{n-1}\left(I^{n}\right)$, найдется функция, интеграл от которой не дифференцируется сильным образом почти всюду на $I^{n}$. Важность этого примера обсуждается, например, в [3], [4]. А. Зигмундом была предложена следующая проблема: можно ли для любой суммируемой на плоскости функции выбрать так оси координат, чтобы интеграл от этой функции был дифференцируем сильным образом?

Отрицательный ответ на поставленньй вопрос был получен Дж. Марстрандом [5]. Он построил пример суммируемой на $I^{2}$ функции, интеграл от которой не дифференцируем сильным образом при любом выборе системы координат. В дальнейшем пример Марстранда модифицировался в [6], [7].

Интересным является тот момент, что мы не располагаем примером функции, интеграл от которой недифференцируем при одном выборе системы координат и дифференцируем при другом. Указанньй пробел и заполняется в настоящей работе.

Теорема. Существует суммируемая функиия, интеграл от которой недиф-

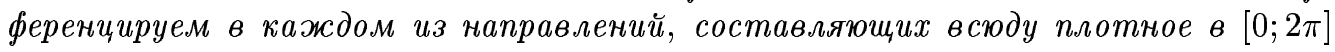
множество, но дифферениируем сильным образом.

Доказательство этой теоремы основывается на следующих леммах.

Пусть $I$ - интервал на прямой длины $a$. Тогда максимальная функция Харди-Литтлвуда от $\chi_{I}(x)$ вычисляется следуюшим образом:

$$
\mathscr{M} \chi_{I}(x)=\frac{a}{\operatorname{dist}(x, I)+a} .
$$

Очевидно, это равенство выполняется и в случае, когда когда $I$ - вертикальная полоса ширины $a$, а вместо максимальной функции Харди-Литтлвуда рассматривается сильная максимальная функция $\mathscr{M}_{s} f$. Возможно, оно справедливо при любом расположении полосы $I$. Во всяком случае предлагаемая ниже лемма 1 содержит близкое к этому утверждение. 
Лемма 1. Пусть П - полоса ширины а. Тогда

$$
\frac{a}{\operatorname{dist}(x, \Pi)+a} \leqslant \mathscr{M}_{s} \chi_{\Pi}(x) \leqslant \frac{a}{\max \{\operatorname{dist}(x, \Pi), a\}} .
$$

ДокАЗАТЕЛЬСТВо. Пусть $R$ - прямоугольник, $x \in R$, а величина $d=O A$ и угол $\theta$ определены на рис. 1.

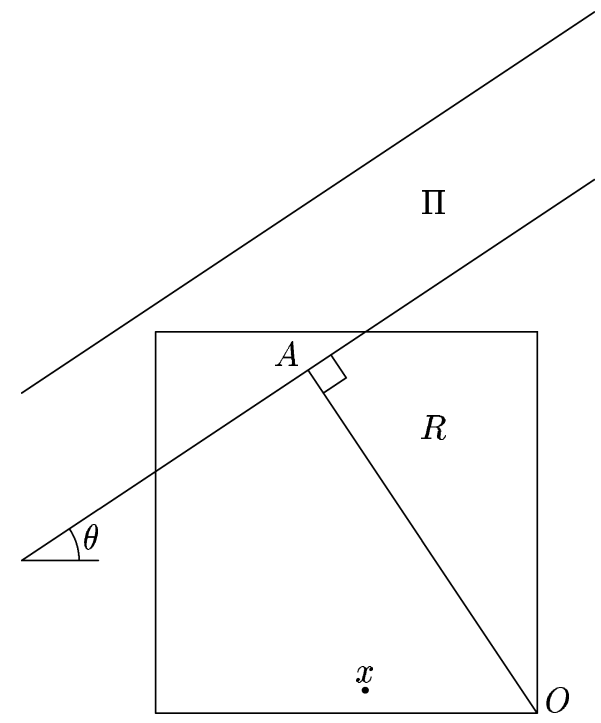

Рис. 1

Если мы покажем, что

$$
\frac{|R \cap \Pi|}{|\Pi|} \leqslant \frac{a}{d}
$$

то поскольку $d \geqslant \operatorname{dist}(x, \Pi)$, правое неравенство будет установлено.

Не ограничивая общности, считаем $0<\theta<\pi / 2, x \notin \Pi$ (см. рис. 1$)$. Пусть $R=I_{1} \times I_{2}$, причем $\left|I_{1}\right|=\alpha,\left|I_{2}\right|=\beta$. Разобьем область параметров $(\alpha, \beta)$ на пять подобластей, как показано на рис. 2.

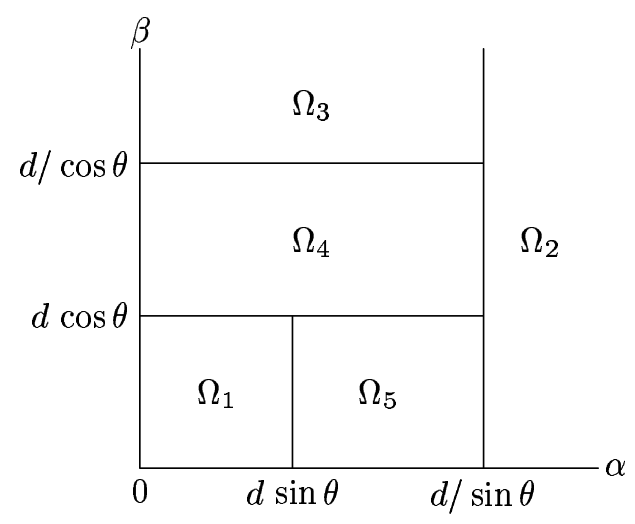

Рис. 2

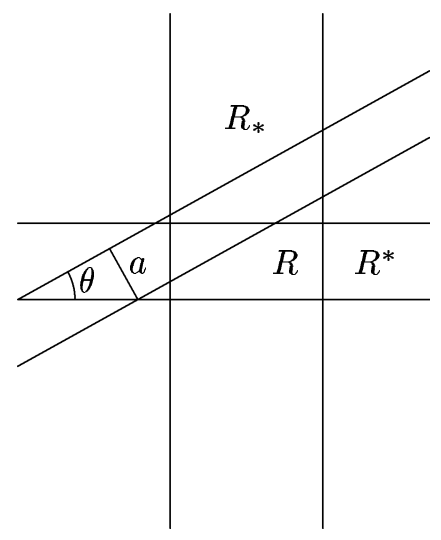

Рис. 3 
1) В случае $(\alpha, \beta) \in \Omega_{1}$ имеем $R \cap \Pi=\varnothing$ и (1) доказано.

2 ) В случае $(\alpha, \beta) \in \Omega_{2}$ имеет место неравенство $\alpha \geqslant d / \sin \theta$. Обозначим через $R_{*}$ и $R^{*}$ вертикальную и горизонтальную полосы соответственно такие, что $R_{*} \cap R^{*}=R$.

Очевидно (см. рис. 3),

$$
\frac{|R \cap \Pi|}{|R|} \leqslant \frac{\left|R^{*} \cap \Pi\right|}{|R|}=\frac{a \beta}{\alpha \beta \sin \theta}=\frac{a}{\alpha \sin \theta} \leqslant \frac{a}{\sin \theta \cdot d / \sin \theta}=\frac{a}{d},
$$

и (1) в этом случае доказано.

3) В случае $(\alpha, \beta) \in \Omega_{3}$ поступаем по аналогии со случаем 2$)$. Так как $\beta \geqslant d / \cos \theta$, имеем

$$
\frac{|R \cap \Pi|}{|R|} \leqslant \frac{\left|R_{*} \cap \Pi\right|}{|R|}=\frac{a \alpha}{\alpha \beta \cos \theta}=\frac{a}{\beta \cos \theta} \leqslant \frac{a}{\cos \theta \cdot d / \cos \theta}=\frac{a}{d},
$$

и (1) в этом случае доказано.

4) В случае $(\alpha, \beta) \in \Omega_{4}$ имеем $d \cos \theta \leqslant \beta \leqslant d / \cos \theta, 0<\alpha \leqslant d / \sin \theta$, откуда (см. рис. 4)

$$
\begin{gathered}
|A B|=\frac{\beta-|A D|}{\sin \theta}, \quad|A D|=\left(\frac{d}{\sin \theta}-\alpha\right) \operatorname{tg} \theta \\
\frac{|\Pi \cap R|}{|R|} \leqslant \frac{|A B| a}{\alpha \beta}=\frac{a}{\alpha \beta \sin \theta}\left(\beta-\left(\frac{d}{\sin \theta}-\alpha\right) \operatorname{tg} \theta\right)=\frac{a}{\alpha \beta \sin \theta}\left(\beta-\frac{d}{\cos \theta}+\alpha \operatorname{tg} \theta\right) \\
=a\left(\frac{1}{\alpha \sin \theta}-\frac{d}{\alpha \beta \sin \theta \cos \theta}+\frac{1}{\beta \cos \theta}\right)=a\left(\frac{1}{\alpha \sin \theta}\left(1-\frac{d}{\beta \cos \theta}\right)+\frac{1}{\beta \cos \theta}\right) .
\end{gathered}
$$

Однако,

$$
1-\frac{d}{\beta \cos \theta} \leqslant 1-\frac{d}{(d / \cos \theta) \cos \theta}=0 .
$$

Поэтому

$$
\frac{|\Pi \cap R|}{|R|} \leqslant a\left(\frac{1}{(d / \sin \theta) \sin \theta}\left(1-\frac{d}{\beta \cos \theta}\right)+\frac{1}{\beta \cos \theta}\right)=a\left(\frac{1}{d}-\frac{1}{\beta \cos \theta}+\frac{1}{\beta \cos \theta}\right)=\frac{a}{d}
$$

и (1) в этом случае доказано.

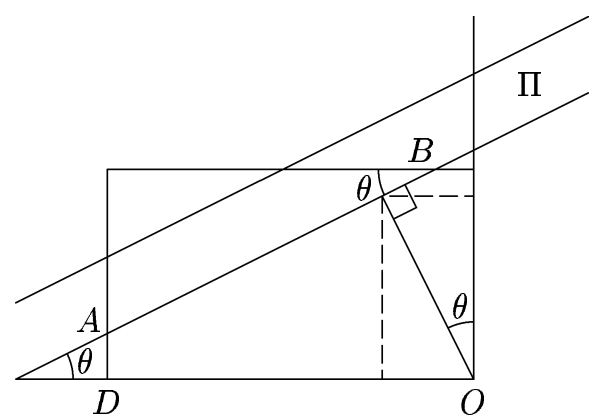

Рис. 4

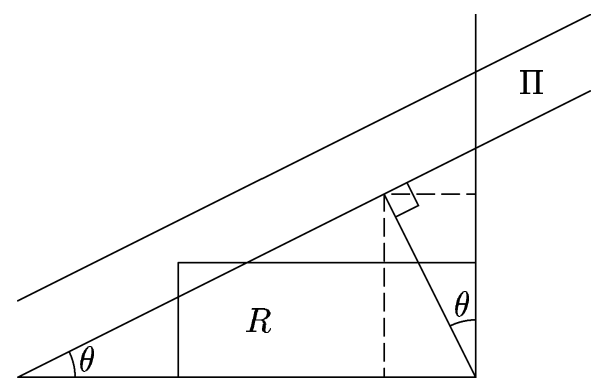

Рис. 5 
5) В случае $(\alpha, \beta) \in \Omega_{5}$ имеем $d \sin \theta<\alpha \leqslant d / \sin \theta, 0<\beta \leqslant d \cos \theta$. Очевидно (см. рис. 5$), \beta \leqslant d / \cos \theta$ и, как и в предыдущем случае, справедлива оценка

$\frac{|\Pi \cap R|}{|R|} \leqslant a\left(\frac{1}{\alpha \sin \theta}-\frac{d}{\alpha \beta \sin \theta \cos \theta}+\frac{1}{\beta \cos \theta}\right)=a\left(\frac{1}{\beta \cos \theta}\left(1-\frac{d}{\alpha \sin \theta}\right)+\frac{1}{\alpha \sin \theta}\right)$.

Однако,

$$
1-\frac{d}{\alpha \sin \theta} \leqslant 1-\frac{d}{(d / \sin \theta) \sin \theta}=0
$$

Поэтому

$\frac{|\Pi \cap R|}{|R|} \leqslant a\left(\frac{1}{(d / \cos \theta) \cos \theta}\left(1-\frac{d}{\alpha \sin \theta}\right)+\frac{1}{\alpha \sin \theta}\right)=a\left(\frac{1}{d}-\frac{1}{\alpha \sin \theta}+\frac{1}{\alpha \sin \theta}\right)=\frac{a}{d}$ и (1) доказано для всех случаев.

Докажем теперь левую часть утверждения леммы 1. Случай $x \in \Pi$ тривиален. В случае $x \notin \Pi$, не ограничивая общности, можно считать $0 \leqslant \theta \leqslant \pi / 4$. Выберем тогда прямоугольник $R$, как показано на рис. 6 .

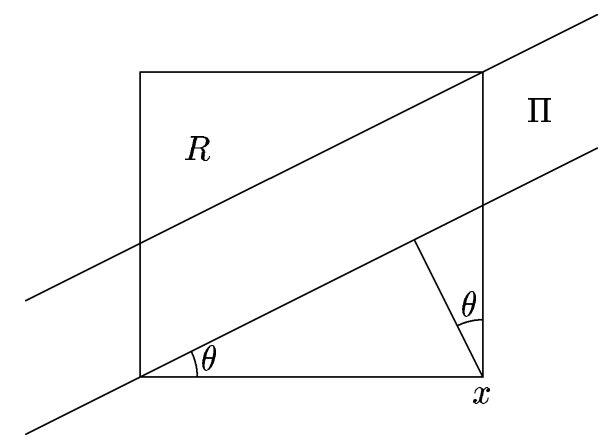

Рис. 6

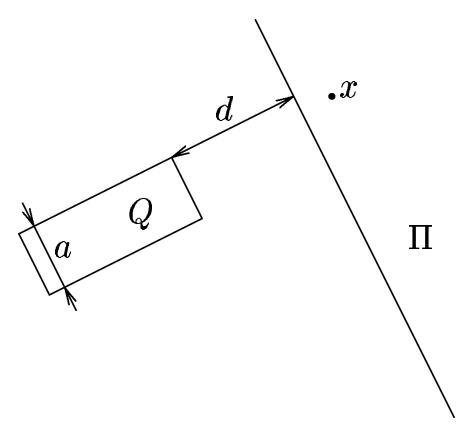

Рис. 7

Тогда

$$
\frac{|\Pi \cap R|}{|R|}=\frac{a \alpha}{\alpha \beta \cos \theta}=\frac{a}{\beta \cos \theta}=\frac{a}{a+d},
$$

что и требовалось доказать.

ЗАмЕчАниЕ 1. В дальнейшем нами будет использоваться только оценка сверху. Оценка снизу включена в лемму 1 для полноты формулировки. Однако, нам представляется достаточно интересным выяснить, можно ли в утверждении леммы 1 вместо левого неравенства писать знак равенства.

Лемма 2. Пусть $Q$ - прямоугольник ширины $а$, наклоненный к оси $O X$ под углом $\theta, 0<\theta<\pi / 2$. Пусть $\Pi-$ полуплоскость, наклоненная $\kappa$ оси $O X$ под углом $\theta+\pi / 2, Q \not \subset \Pi u d \equiv \operatorname{dist}(Q, \Pi) \geqslant a($ cм. puc. 7$)$.

Тогда для всех $x \in \Pi$ справедлива следующая оченка для сильного максимального оператора:

$$
\mathscr{M}_{s} \chi_{Q}(x) \leqslant \frac{2 a}{d \sin \theta \cos \theta} .
$$




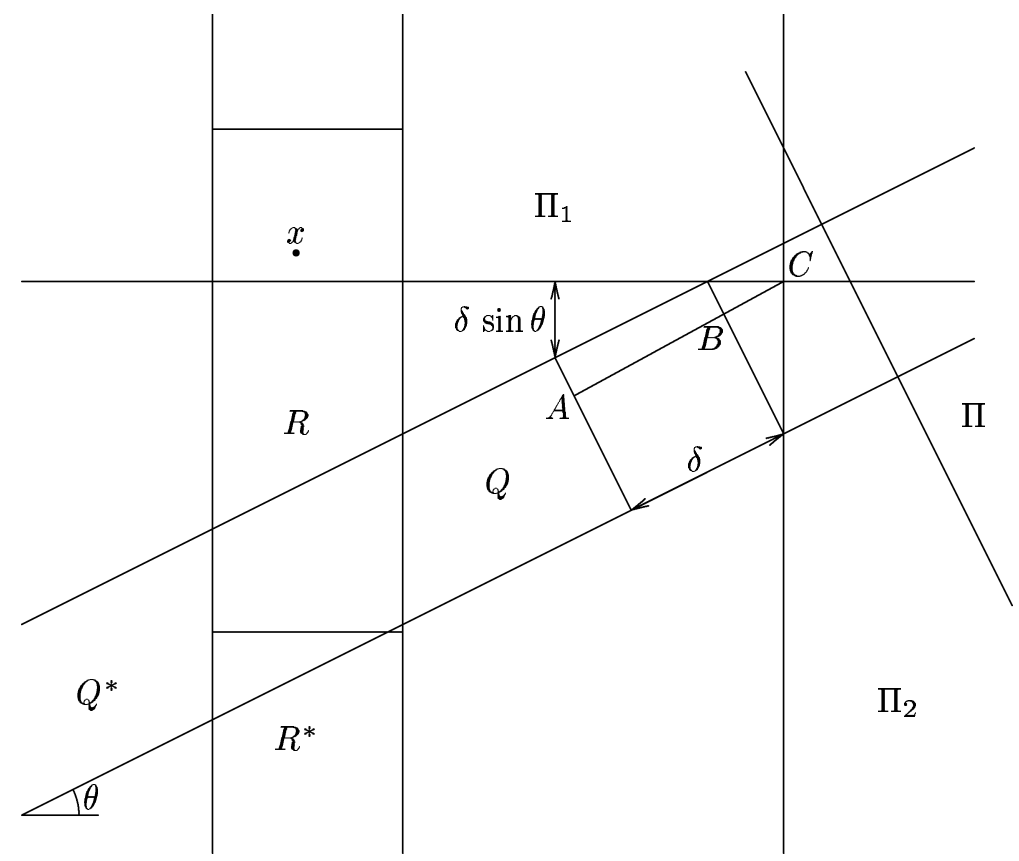

Рис. 8

ДокАЗАТЕЛЬСТво. Положим $\delta=d-a \sin \theta \cos \theta$ и проведем взаимноортогональные полуплоскости $\Pi_{1}$ и $\Pi_{2}$, не содержащие $Q$, как показано на рис. 8 .

Пусть $x \in \Pi_{1}, R$-прямоугольник, содержащий $x$ и имеющий размеры $\alpha \times \beta$. Тогда $\beta \geqslant \delta \sin \theta$ и если $R^{*}$ - вертикальная полоса, порожденная $R$, а $Q^{*}$ - полоса, порожденная $Q$ (см. рис. 8 ), то

$$
\begin{aligned}
\frac{|Q \cap R|}{|R|} & \leqslant \frac{\left|Q^{*} \cap R^{*}\right|}{|R|}=\frac{(a / \cos \theta) \alpha}{\alpha \beta}=\frac{a}{\beta \cos \theta} \leqslant \frac{a}{\delta \sin \theta \cos \theta} \\
& \leqslant \frac{a /(\sin \theta \cos \theta)}{d-a \sin \theta \cos \theta}=\frac{a /(\sin \theta \cos \theta)}{d-(a / 2) \sin (2 \theta)} \leqslant \frac{a /(\sin \theta \cos \theta)}{d-a / 2} \leqslant \frac{2 a}{d \sin \theta \cos \theta} .
\end{aligned}
$$

Аналогичную оценку можно получить для $x \in \Pi_{2}$.

Покажем теперь, что $\Pi \subset \Pi_{1} \cap \Pi_{2}$, для чего нужно вычислить расстояние $|A C|$ от $Q$ до точки $A$ пересечения границ полуплоскостей $\Pi_{1}$ и $\Pi_{2}$. Имеем $|A C|=|A B|+|B C|=$ $\delta+|B C|$. Однако, $|B C|=a \sin \theta \cos \theta$. Поэтому $|A C|=\delta+a \sin \theta \cos \theta=d$, что и доказывает лемму 2.

Следующая лемма В.Г. Спринжука является обобщением леммы Бореля-Кантелли (см. $[8$, гл. $1, \S 3$, лемма 5$])$.

Лемма 3. Пусть $(\Omega, \mathscr{A}, \mu)$ - пространство с мерой и для последовательности $A_{k} \in \mathscr{A}$

$$
\sum_{k=1}^{\infty} \mu\left(A_{k}\right)=\infty
$$

Тогда

$$
\mu\left(\overline{\lim } A_{k}\right) \geqslant \varlimsup_{q \rightarrow \infty} \frac{\left(\sum_{k=1}^{q} \mu\left(A_{k}\right)\right)^{2}}{\sum_{k, j=1}^{q} \mu\left(A_{k} \cap A_{j}\right)} .
$$


Приступим теперь непосредственно к доказательству теоремы.

ДокАЗАТЕЛЬСТво тЕоремЫ. Определим натуральные числа $\lambda_{k} \uparrow \infty$ так, чтобы для $B_{k} \equiv \lambda_{k}^{-1}$

$$
\sum_{k=1}^{\infty} B_{k}^{2} \leqslant \frac{1}{64}, \quad \sum_{k=1}^{\infty} B_{k}^{2} \log \frac{1}{B_{k}}=\infty .
$$

Кроме того, выберем натуральные числа $w_{k} \uparrow \infty$ так, чтобы для $q_{k}=2^{w_{k}}$

$$
\sum_{k=1}^{\infty} B_{k}^{3} q_{k} \leqslant \frac{1}{4}, \quad \sum_{k=1}^{\infty} B_{k}^{2} q_{k}^{2}<\infty
$$

и положим $A_{k}=B_{k}^{2}$.

Введем двоично-рациональные направления

$$
\theta_{j}^{k}=\frac{\pi j}{2 q_{k}}, \quad j=1, \ldots, q_{k}-1
$$

Определим теперь прямоугольники $Q_{j}^{k, *}$ и $\Pi_{j}^{k, *}$ с центрами в начале координат и попарно параллельными сторонами размера $B_{k} \times A_{k}$ и $\left(B_{k}+2 B_{k} q_{k}^{2}\right) \times\left(A_{k}+2 B_{k} q_{k}\right)$ соответственно, большие стороны которых составляют с осью $O X$ угол $\theta_{j}^{k}$.

В силу соотношения (3)

$$
\sum_{k=1}^{\infty} \sum_{j=1}^{q_{k}}\left|\Pi_{j}^{k, *}\right| \ll \sum_{k=1}^{\infty} B_{k}^{2} q_{k}^{3}<\infty .
$$

Здесь и далее запись $x \ll y$ трактуется как неравенство $x \leqslant c y$, справедливое с некоторой константой $c$.

Определим теперь фигуру $Y_{j}^{k, *}$, совпадающую после поворота осей координат на угол $\theta_{j}^{k}$ против часовой стрелки и сдвига на величину $B_{k} / 2$ в положительном направлении оси $O X$ и на величину $A_{k} / 2$ в положительном направлении оси $O Y$ с криволинейной трапецией $Y_{k}^{*}$, ограниченной гиперболой $x_{2}=A_{k} / x_{1}$, осью $O X$ и вертикальными прямыми $x_{1}=B_{k}$ и $x_{1}=1 / 4$ (см. рис. 9 ).

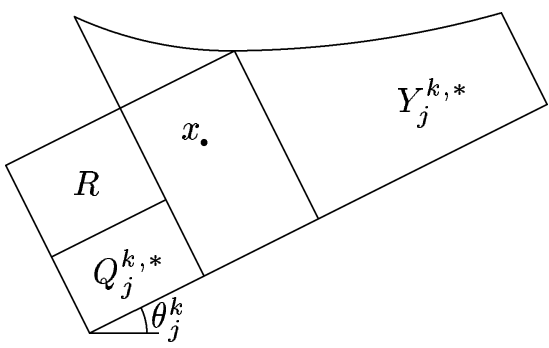

Рис. 9

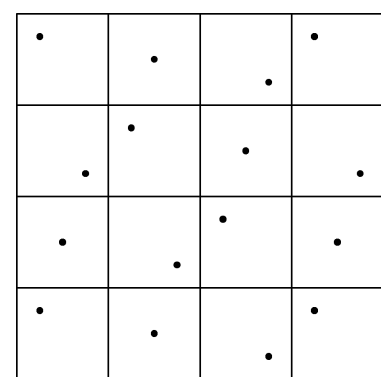

Рис. 10 
Легко видеть, что $Y_{j}^{k, *}$ содержится в круге с центром в начале координат радиуса $1 / 2$ и для любого $j$

$$
\left|Y_{j}^{k, *}\right|=\left|Y_{k}^{*}\right|=\int_{B_{k}}^{1 / 4} \frac{A_{k}}{t} d t \geqslant \frac{1}{4} A_{k} \log \frac{1}{B_{k}} .
$$

Зафиксируем теперь направление $\theta_{r}^{\nu}$ и положим $\theta_{j}^{k}=\theta_{r}^{\nu}$. Тогда множества $Y_{j}^{k, *}$ представляют собой криволинейные трапеции, ограниченные гиперболами с убьвающими коэффициентами и повернутыми на один и тот же угол $\theta_{j}^{k}$, причем с учетом (2)

$$
\sum_{k=\nu}^{\infty}\left|Y_{j}^{k, *}\right|=\infty
$$

Функцию $f$ будем строить, разбивая $I^{2}$ на $k$-м шаге на $m_{k}^{2}$ одинаковых квадратов $I_{n}^{k}$, $n=1, \ldots, m_{k}^{2}$. В каждом $I_{n}^{k}$ определим некоторую точку $c_{n}^{k}=\left(c_{n, 1}^{k}, c_{n, 2}^{k}\right)$, расположенную на диагонали $I_{n}^{k}$ (выбор $c_{n}^{k}$ будет определен ниже), и будем помещать прямоугольники $Q_{n, j}^{k}$ и $\Pi_{n, j}^{k}$ с центрами в точках $c_{n}^{k}$ и со сторонами, наклоненными к осям координат на угол $\theta_{j}^{k}$, размера $B_{k} / m_{k} \times A_{k} / m_{k}$ и $\left(B_{k}+2 B_{k} q_{k}^{2}\right) / m_{k} \times\left(A_{k}+2 B_{k} q_{k}\right) / m_{k}$ соответственно.

Покажем теперь, как выбираются числа $m_{k}$ и точки $c_{n}^{k}$. Обозначим через $H_{n}^{k}$ гомотетию с коэффициентом $1 / m_{k}$, переводящую начало координат в точку $c_{n}^{k}$.

В этих терминах прямоугольники $Q_{n, j}^{k}$ и $\Pi_{n, j}^{k}$ можно определить следуюшим образом:

$$
Q_{n, j}^{k}=H_{n}^{k}\left(Q_{j}^{k, *}\right), \quad \Pi_{n, j}^{k}=H_{n}^{k}\left(\Pi_{j}^{k, *}\right) .
$$

Определим также следующие множества:

$$
\begin{gathered}
Q_{n}^{k} \equiv \bigcup_{j=1}^{q_{k}} Q_{n, j}^{k}, \quad Q^{k} \equiv \bigcup_{n=1}^{m_{k}^{2}} Q_{n}^{k}, \quad \Pi_{n}^{k} \equiv \bigcup_{j=1}^{q_{k}} \Pi_{n, j}^{k}, \quad \Pi^{k} \equiv \bigcup_{n=1}^{m_{k}^{2}} \Pi_{n}^{k}, \\
Y_{n, j}^{k} \equiv H_{n}^{k}\left(Y_{j}^{k, *}\right), \quad Y_{n}^{k} \equiv \bigcup_{j=1}^{q_{k}} Y_{j}^{k}, \quad Y^{k} \equiv \bigcup_{n=1}^{m_{k}^{2}} Y_{n}^{k} .
\end{gathered}
$$

В силу (4)

$$
\sum_{k=1}^{\infty}\left|\Pi^{k}\right|=\sum_{k=1}^{\infty}\left|\Pi_{k}^{*}\right|<\infty
$$

Теперь на $k$-м шаге будут появляться в рассмотрении $q_{k}$ двоично-рациональных направлений $\theta_{j}^{k}$. Для $k=1$ положим $m_{1}=1, c_{1}^{1}=(1 / 2,1 / 2), Q_{1}=Q^{1}, Y_{1}=Y^{1}, J_{1}=\{1\}$.

Пусть числа $m_{1}, \ldots, m_{k}$ уже выбраны так, чтобы для любого направления $\theta_{r}^{\nu}=$ $\pi r /\left(2 q_{\nu}\right)$, где $r / q_{\nu}$ - несократимая дробь, $1 \leqslant \nu \leqslant k-1$, вьполнялось следующее свойство: если $\nu \leqslant l, s \leqslant k, \theta_{j}^{s}=\theta_{i}^{l}=\theta_{r}^{\nu}$, то

$$
\left|Y_{j}^{s} \cap Y_{i}^{l}\right| \leqslant 2\left|Y_{j}^{s}\right| \cdot\left|Y_{i}^{l}\right|
$$


Пусть $\nu \leqslant s \leqslant k, a_{s, j}=\left\{n: I_{n}^{k+1} \cap Y_{j}^{s} \neq \varnothing\right\}$. Запись $a^{\# ~ з д е с ь ~ и ~ д а л е е ~ о з н а ч а е т ~}$ число элементов множества $a$. Так как $Y_{j}^{s}$ измеримо по Жордану, имеем

$$
\lim _{\operatorname{diam} I_{n}^{k+1} \rightarrow 0}\left|\bigcup_{n \in a_{s, j}} I_{n}^{k+1}\right|=\left|Y_{j}^{s}\right|
$$

Следовательно, существует $m_{s, j}$ такое, что

$$
a_{s, j}^{\#}\left|I_{n}^{k+1}\right|=\left|\bigcup_{n \in a_{s, j}} I_{n}^{k+1}\right| \leqslant 2\left|Y_{j}^{s}\right|
$$

Положим теперь

$$
m_{k+1}^{*}=\max \left\{m_{s, j}: 1 \leqslant s \leqslant k, 1 \leqslant j \leqslant q_{s}\right\}, \quad m_{k+1}=\max \left\{m_{k+1}^{*}, 4 \lambda_{k} m_{k}\right\} .
$$

Тогда для указанного вьше направления $\theta_{r}^{\nu}, 1 \leqslant \nu \leqslant k$, и $\theta_{j}^{k+1}=\theta_{i}^{s}=\theta_{r}^{\nu}$ при любом выборе точек $c_{n}^{k+1}$

$$
\left|Y_{i}^{s} \cap Y_{j}^{k+1}\right| \leqslant\left|\bigcup_{n \in a_{s, j}} Y_{j, n}^{k+1}\right|=a_{s, j}^{\#}\left|Y_{j, n}^{k+1}\right| \leqslant 2 \frac{\left|Y_{j}^{s}\right|}{\left|I_{n}^{k+1}\right|}\left|Y_{j, n}^{k+1}\right|=2\left|Y_{j}^{s}\right| \cdot\left|Y_{j}^{k+1}\right| .
$$

Таким образом, (7) вьполнено с заменой $k$ на $k+1$.

Выбирая в случае необходимости рост чисел $m_{k}$ более быстрым, потребуем, чтобы

$$
\left|\bigcup_{s \in J_{k}} Y_{s}^{k+1}\right| \leqslant 2^{-k}
$$

где

$$
J_{k}=\left\{n: 3 \cdot I_{n}^{k+1} \cap\left(\bigcup_{l=1}^{k} \partial Q_{l} \cup \bigcup_{l=1}^{k} \partial Y_{l}\right) \neq \varnothing\right\}
$$

а $3 \cdot I$ обозначает концентрический с $I$ квадрат, $|3 \cdot I|=3|I|$.

Кроме того, будем считать $m_{k}$ кратньпи $\lambda_{k}$.

Определим теперь точки $c_{n}^{k}$ внутри

$$
I_{n}^{k}=\left[\frac{s-1}{m_{k}}, \frac{s}{m_{k}}\right] \times\left[\frac{i-1}{m_{k}}, \frac{i}{m_{k}}\right]
$$

для $i=1$. Положим для $s \leqslant \lambda_{k}$

$$
c_{n, 1}^{k}=\frac{s-1}{m_{k}}+\frac{1}{\lambda_{k} m_{k}} \frac{2 s-1}{2}, \quad c_{n, 2}^{k}=\frac{1}{m_{k}}-\frac{1}{\lambda_{k} m_{k}} \frac{2 s-1}{2} .
$$

Таким образом, выбранная точка $c_{n}^{k}$ расположена на диагонали $I_{n}^{k}$. Покажем, что $c_{n}^{k} \in I_{n}^{k}$. Действительно,

$$
\frac{s-1}{m_{2}} \leqslant c_{n, 1}^{k} \leqslant \frac{s-1}{m_{2}}+\frac{1}{\lambda_{k} m_{k}} s \leqslant \frac{s-1}{m_{2}}+\frac{1}{m_{k}}=\frac{s}{m_{2}},
$$


что и требовалось показать.

Отметим, что мы определили положение точек внутри $\lambda_{k}$ квадратов из нижнего слоя разложения $I^{2}$. Определим точки $c_{n}^{k}$ внутри остальных квадратов из нижнего слоя путем элементарного дублирования их расположения в первых $\lambda_{k}$ квадратах. При этом

$$
c_{n, 1}^{k} \equiv c_{s, 1}^{k}\left(\bmod \frac{1}{\lambda_{k} m_{k}}\right)
$$

и, кроме того, если $c_{n, 2}^{k}=c_{s, 2}^{k}$, то

$$
c_{n, 1}^{k} \equiv c_{s, 1}^{k}\left(\bmod \frac{\lambda_{k}}{m_{k}}\right)
$$

Во втором слое расположим нижний слой с циклическим сдвигом на один квадрат влево, в третьем слое расположим предыдущий слой с циклическим сдвигом на один квадрат влево, и т.д. На каждом следующем слое будем располагать предыдущий с циклическим сдвигом на один квадрат (см. рис. 10). Тогда соотношение (9) останется в силе и, более того, для точек $c_{n}^{k}$, у которых $c_{n, 1}^{k}=c_{s, 1}^{k}$, выполнено

$$
c_{n, 2}^{k} \equiv c_{s, 2}^{k}\left(\bmod \frac{\lambda_{k}}{m_{k}}\right)
$$

Теперь для любого направления $\theta_{r}^{\nu}=\pi r /\left(2 q_{\nu}\right)$, где $r / q_{\nu}-$ несократимая дробь, а $\theta_{j}^{k}=\theta_{r}^{\nu}$, с учетом гомотетичности, соотношения (5) и леммы 3 имеем

$$
\left|\varlimsup Y_{j}^{k}\right| \geqslant \frac{1}{2}
$$

Определим функции

$$
f_{n, j}^{k}=\lambda_{k} \chi_{Q_{n, j}^{k}}, \quad f_{n}^{k}=\sum_{j=1}^{q_{k}-1} f_{n, j}^{k}, \quad f^{k}=\sum_{n=1}^{m_{k}^{2}} f_{n}^{k}, \quad f=\sum_{k=1}^{\infty} f^{k} .
$$

Проверим суммируемость $f$. С учетом (3) имеем

$$
\|f\|_{1}=\sum_{k=1}^{\infty} \sum_{n=1}^{m_{k}^{2}} \sum_{j=1}^{q_{k}-1} \lambda_{k}\left|Q_{n, j}^{k}\right| \leqslant \sum_{k=1}^{\infty} \lambda_{k} m_{k}^{2} q_{k} B_{k} A_{k} m_{k}^{-2}=\sum_{k=1}^{\infty} q_{k} B_{k}^{2}<\infty
$$

Покажем теперь недифференцируемость интеграла от $f$ базисом из прямоугольников в направлении $\theta_{j}^{k}$.

Если $x \in Y_{j, n}^{k}$, то всегда найдется прямоугольник $R \ni x$ такой, что $|R|=A_{k} / m_{k}^{2}$, $Q_{n, j}^{k} \subset R$ (см. рис. 9). Тогда

$$
|R|^{-1} \int_{R} f_{j, n}^{k}(y) d y=\lambda_{k}\left|Q_{n, j}^{k}\right| \frac{m_{k}^{2}}{A_{k}}=\lambda_{k} \frac{A_{k} B_{k}}{m_{k}^{2}} \frac{m_{k}^{2}}{A_{k}}=1
$$


Таким образом, с учетом (12) на множестве меры больше $1 / 2$ верхняя производная интеграла относительно указанного базиса не меньше 1.

С другой стороны, если $\operatorname{supp} \varphi \equiv\{x: \varphi(x) \neq 0\}$, то

$$
|\operatorname{supp} f| \leqslant \sum_{k=1}^{\infty}\left|\operatorname{supp} f_{k}\right| \leqslant \sum_{k=1}^{\infty} A_{k} B_{k} q_{k}=\sum_{k=1}^{\infty} B_{k}^{3} q_{k}<\frac{1}{4}
$$

в силу (3).

Сопоставляя (13) и (14), получаем требуемую недифференцируемость.

После того, как мы установили недифференцируемость интеграла от $f$ в каждом двоично-рациональном направлении, покажем сильную дифференцируемость. В силу теоремы А. С. Безиковича (см., например, [4]) достаточно показать конечность почти всюду верхней сильной производной интеграла от $f$, т.е. установить, что для почти всех $x$

$$
|R|^{-1} \int_{R \ni x} f(y) d y=O_{x}(1), \quad \operatorname{diam}(R) \rightarrow 0 .
$$

С этой целью нужно накопить множество $E$ нулевой меры, на дополнении к которому будет справедливо (15). Стандартная техника теории функций позволяет накапливать такие множества, используя свойства верхнего предела множеств и лемму Бореля-Кантелли.

А именно, в силу выбора $m_{k}$ мера тех $3 \cdot I_{j}^{k}$, которые покрьвают границу $\bigcup_{n=1}^{k-1} Q^{n}$, будет меньше чем $2^{-k}$ (см. (8)). Следовательно, по лемме Бореля-Кантелли верхний предел этих $I_{j}^{k}$ (обозначим его через $E_{1}$ ) имеет меру 0.

Далее, пусть $E_{2}=\varlimsup_{n} \Pi_{n}$. С учетом (6) и леммы Бореля-Кантелли $\left|E_{2}\right|=0$. Положим, наконец, $E=E_{1} \cup E_{1},|E|=0$, и ниже будем предполагать, что $x \notin E$.

Тогда найдется номер $n^{*}(x)$ такой, что

$$
3 \cdot I_{j}^{k} \cap \bigcup_{n=1}^{k-1} \partial Q^{n}=\varnothing \quad \text { для всех } I_{j}^{k} \ni x, \quad k>n^{*}(x) .
$$

Кроме того, существует номер $n(x)$ такой, что

$$
x \notin \Pi_{k} \quad \text { для всех } k \geqslant n(x) .
$$

Пусть $x \in R=\alpha \times \beta, \alpha \geqslant \beta$. Определим $k \leqslant s$ так, чтобы

$$
m_{k+1}^{-1}<\alpha \leqslant m_{k}^{-1}, \quad m_{s+1}^{-1}<\beta \leqslant m_{s}^{-1} \text {. }
$$

Будем рассматривать $R$ настолько маленьким, чтобы $k \geqslant n(x)+n^{*}(x)$, и считать $x \in I_{j}^{k}$.

Имеем

$$
\begin{aligned}
|R|^{-1} \int_{R \ni x} f(y) d y= & \sum_{n=1}^{k-1}|R|^{-1} \int_{R \ni x} f^{n}(y) d y+|R|^{-1} \int_{R \ni x} f^{n}(y) d y \\
& +\sum_{n=k+1}^{s-1}|R|^{-1} \int_{R \ni x} f^{n}(y) d y+|R|^{-1} \int_{R \ni x} f^{s}(y) d y \\
& +|R|^{-1} \int_{R \ni x} \sum_{n=s+1}^{\infty} f^{n}(y) d y \\
\equiv & S_{1}+S_{2}+S_{3}+S_{4}+S_{5} .
\end{aligned}
$$


Так как $k \geqslant n^{*}(x)$ и $x \in I_{j}^{k}$, для $1 \leqslant n \leqslant k-1$ в силу (16) либо $3 \cdot I_{j}^{k} \subset Q^{n}$, либо $3 \cdot I_{j}^{k} \cap Q^{n}=\varnothing$. Учитьвая, что $R \subset 3 \cdot I_{j}^{k}$, получим

$$
S_{1} \leqslant \sum_{n=1}^{k-1}|R|^{-1} \int_{R \ni x} f^{n}(y) d y \leqslant \sum_{n=1}^{k-1} f^{n}(x) \leqslant f(x)=O_{x}(1) .
$$

Оценим теперь $S_{2}$. Поскольку $R$ может пересекаться не более чем с четырьмя из квадратов $I_{n}^{k}$, не ограничивая обшности, считаем

$$
S_{2} \leqslant 4 \lambda_{k} \sum_{j=1}^{q_{k}-1}|R|^{-1} \int_{R \ni x} \chi_{Q_{n, j}^{k}}(y) d y \leqslant 4 \lambda_{k} \sum_{j=1}^{q_{k}-1} \mathscr{M}_{s} \chi_{Q_{n, j}^{k}}(x),
$$

где $\mathscr{M}_{s}$, как и ранее, обозначает сильную максимальную функцию.

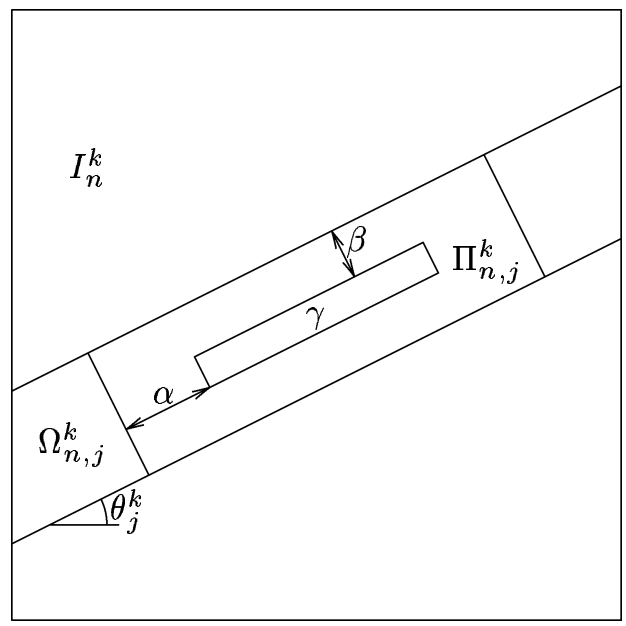

Рис. 11

Обозначим через $\Omega_{n, j}^{k}$ горизонтальную полосу, порожденную продолжением сторон $\Pi_{n, j}^{k}$ (см. рис. 11, где положено $\alpha=B_{k} q_{k}^{2} / m_{k}, \beta=B_{k} q_{k} / m_{k}, \gamma=Q_{n, j}^{k}$ ). С учетом (17) возможны два случая расположения точки $x$ :

1) $x \in I_{n}^{k} \backslash \Omega_{n, j}^{k}$;

2) $x \in \Omega_{n, j}^{k} \backslash \Pi_{n, j}^{k}$.

1) Применяя лемму 1, получим

$$
\mathscr{M}_{s} \chi_{Q_{n, j}^{k}}(x) \leqslant \frac{A_{k} m_{k}}{B_{k} q_{k} m_{k}}=\frac{A_{k}}{B_{k} q_{k}}=\frac{B_{k}}{q_{k}}=\frac{1}{\lambda_{k} q_{k}} .
$$

Следовательно, $S_{2} \leqslant 4$.

2) Применяя лемму 2, получим

$$
\mathscr{M}_{s} \chi_{Q_{n, j}^{k}}(x) \ll \frac{A_{k} m_{k}}{q_{k}^{2} \theta_{j}^{k} B_{k} m_{k}} \ll \frac{B_{k}}{q_{k}}=\frac{1}{\lambda_{k} q_{k}} .
$$


Заметим, что в этом месте доказательства существенно использовался тот факт, что множество направлений дифференцируемости $\theta_{j}^{k}$ отделено от нуля величиной $q_{k}^{-1}$.

В итоге $S_{2}=O(1)$.

Оценим теперь $S_{3}$.

Так как в силу выбора $m_{k}$ справедливо неравенство $m_{s} \geqslant 4 m_{s-1} \lambda_{s-1}$, согласно (9) имеем

$$
\beta \leqslant m_{s}^{-1} \leqslant\left(4 m_{s-1} \lambda_{s-1}\right)^{-1} \leqslant\left(4 m_{l} \lambda_{l}\right)^{-1} \leqslant \frac{1}{4}\left|c_{n, 2}^{l}-c_{r, 2}^{l}\right| \equiv \gamma
$$

для любых $n, r=1, \ldots, m_{l}^{2}$. Иньми словами, расстояние между центрами любых $Q_{n}^{l}$ больше четырех величин высоты прямоугольника $R$ (см. рис. 12 ).

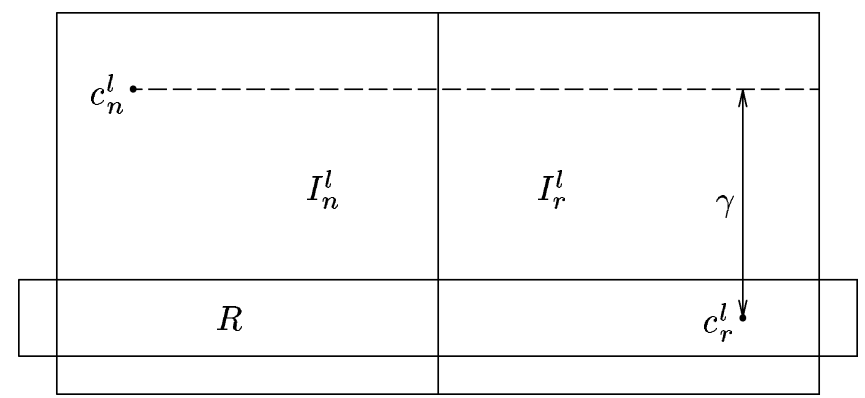

Рис. 12

Таким образом, если $c_{n}^{l} \subset 3 \cdot R$ и $c_{r}^{l} \subset 3 \cdot R$, то в силу (18)

$$
c_{n, 1}^{l}=c_{r, 1}^{l}
$$

Пусть $\Gamma_{l}=\left\{n: c_{n}^{l} \subset 3 \cdot R\right\}$. Тогда из (19) и (11) следует, что число элементов $\Gamma_{l}$ удовлетворяет оценке

$$
\Gamma_{l}^{\#} \leqslant \frac{\alpha}{\lambda_{l} / m_{l}}=\frac{|R| m_{l}}{\beta \lambda_{l}} .
$$

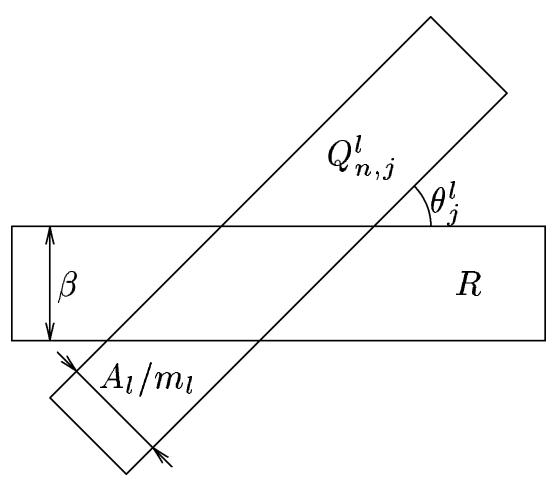

Рис. 13 
Следовательно,

$$
|R|^{-1} \int_{R} f^{l}(y) d y \leqslant \sum_{n \in \Gamma_{l}}|R|^{-1} \int_{R} f_{n}^{l}(y) d y \leqslant \sum_{n \in \Gamma_{l}} \lambda_{l} \frac{\left|Q_{n}^{l} \cap R\right|}{|R|} .
$$

Чтобы оценить $\left|Q_{n}^{l} \cap R\right|$, рассмотрим рис. 13. Тогда

$$
\left|Q_{n}^{l} \cap R\right| \leqslant \sum_{j=1}^{q_{l}-1}\left|Q_{n, j}^{l} \cap R\right| \ll \sum_{j=1}^{q_{l}-1} \beta \frac{A_{l}}{m_{l} \theta_{j}^{l}} \leqslant \sum_{j=1}^{q_{l}-1} \frac{\beta A_{l} q_{l}}{m_{l}} \leqslant \frac{\beta A_{l} q_{l}^{2}}{m_{l}} .
$$

Итак, для $1 \leqslant l \leqslant s-1$ имеем

Отсюда в силу (3)

$$
|R|^{-1} \int_{R} f^{l}(y) d y \leqslant \frac{|R| m_{l}}{\beta \lambda_{l}} \lambda_{l} \frac{\beta A_{l} q_{l}^{2}}{m_{l}} \frac{1}{|R|}=A_{l} q_{l}^{2} .
$$

$$
S_{3} \ll \sum_{l=1}^{\infty} A_{l} q_{l}^{2}=\sum_{l=1}^{\infty} B_{l}^{2} q_{l}^{2}=O(1)
$$

Оценим теперь $S_{4}$.

Введем горизонтальные полосы

$$
\Omega_{i}^{s}=R \times\left[\frac{i-1}{m_{s} \lambda_{s}}, \frac{i}{m_{s} \lambda_{s}}\right] .
$$

Пусть на этот раз $\Gamma_{s}=\left\{i: \Omega_{i}^{s} \cap 3 \cdot R \neq \varnothing\right\}$. Очевидно, что количество элементов $\Gamma_{s}$ удовлетворяет оценке

$$
\Gamma_{s}^{\#} \leqslant\left[3 \beta m_{s} \lambda_{s}\right]+2
$$

Обозначим $\Gamma_{s, i}=\left\{n: c_{n}^{s} \subset\left(R \cap \Omega_{i}^{s}\right)\right\}$. Тогда в силу (10)

$$
\Gamma_{s, i}^{\#} \leqslant \frac{3 \alpha}{\lambda_{s} / m_{s}}=\frac{3 m_{s}|R|}{\lambda_{s} \beta} .
$$

Если $\left[3 \beta m_{s} \lambda_{s}\right]>0$, то $\Gamma_{s}^{\#} \leqslant 9 \beta m_{s} \lambda_{s}$. В этом случае внутри $R$ целиком помещается относительно много $Q_{n}^{s}$. Тогда оцениваемые средние мало отличаются от средних по всему $I^{2}$ и вьполнено

$$
\begin{aligned}
|R|^{-1} \int_{R} f^{s}(y) d y & \leqslant \sum_{i \in \Gamma_{s}} \sum_{n \in \Gamma_{s, i}}|R|^{-1} \int_{R} f_{n}^{s}(y) d y \ll \sum_{i \in \Gamma_{s}} \sum_{n \in \Gamma_{s, i}}|R|^{-1} \lambda_{s}\left|Q_{n}^{s}\right| \\
& \leqslant \frac{\Gamma_{s}^{\#} \Gamma_{s, i}^{\#} \lambda_{s} q_{s} A_{s} B_{s}}{|R| m_{s}^{2}} \ll \frac{\beta m_{s} \lambda_{s} m_{s}|R| \lambda_{s} q_{s} A_{s} B_{s}}{\lambda_{s} \beta|R| m_{s}^{2}}=q_{s} A_{s} .
\end{aligned}
$$

Таким образом, если $\left[3 \beta m_{s} \lambda_{s}\right]>0$, то

$$
|R|^{-1} \int_{R} f^{s}(y) d y \ll q_{s} A_{s} .
$$

Если же $0<3 \beta m_{s} \lambda_{s}<1$, т.е. $R$ - очень узкий прямоугольник (см. рис. 14 ), то учитывая, что $\Gamma_{s}^{\#} \leqslant 2$, и соотношение $(20)$, получим

$$
\begin{aligned}
& |R|^{-1} \int_{R} f^{s}(y) d y \leqslant 2 \frac{3 m_{s}|R|}{\lambda_{s} \beta} \frac{\lambda_{s} A_{s} q_{s}^{2}}{m_{s} \alpha}=6 q_{s}^{2} A_{s} . \\
& \overrightarrow{0} \frac{\dot{1}}{m_{s+1}} \quad \vec{\beta} \quad \frac{\dot{1}}{m_{s} \lambda_{s}} \quad \frac{1}{m_{s}}
\end{aligned}
$$

Рис. 14 
В итоге $S_{4}=O(1)$.

Оценим теперь $S_{5}$. Пусть

$$
\Gamma_{l}=\left\{n: I_{n}^{l} \subset 3 \cdot R\right\}, \quad R_{0}(l)=\bigcup_{n \in \Gamma_{l}} I_{n}^{l} .
$$

Для $l \geqslant s+1$ имеем $|R| \leqslant\left|R_{0}(l)\right| \leqslant 9|R|$. Следовательно, для $M \geqslant s+1$ вьполнено

$$
|R|^{-1} \int_{R_{l}} \sum_{l=s+1}^{M} f^{l}(y) d y \leqslant \sum_{l=s+1}^{M} 9\left|R_{0}(l)\right|^{-1} \int_{R_{0}(l)} f^{l}(y) d y \leqslant \sum_{l=s+1}^{M} 9\left\|f^{l}\right\|_{1} \leqslant 9 \sum_{l=1}^{\infty}\left\|f^{l}\right\|_{1} .
$$

Устремляя теперь $M \rightarrow \infty$, по лемме Фату получим

$$
S_{5} \leqslant 9 \sum_{l=1}^{\infty}\left\|f^{l}\right\|_{1}
$$

В итоге $S_{5}=O(1)$.

Следовательно, соотношение (15) установлено, что влечет сильную дифференцируемость интеграла от $f$.

Теорема доказана.

ЗАмЕчАниЕ 2. Было бы интересным выяснить, каким может быть множество направлений дифференцируемости. В частности, может ли это множество быть произвольным подмножеством $[0,2 \pi]$ или же быть дополнением к $\{0, \pi / 2, \pi, 3 \pi / 2\}$ ?

\section{СПИСОК ЦИТИРОВАННОЙ ЛИТЕРАТУРЫ}

[1] Jessen B., Marcinkiewicz J., Zygmund A. Note on the differentiability of multiple integrals // Fund. Math. 1935. V. 25. P. 217-234.

[2] Saks S. On the strong derivatives of functions of intervals // Fund. Math. 1935. V. 25. P. 235-252.

[3] Зигмунд А. Тригонометрические ряды. М.: Мир, 1965.

[4] Гусман М. Дифференцирование интегралов в $\mathbb{R}^{n}$. М.: Мир, 1978.

[5] Marstrand J. A counter-examplein the theory of strong differentiation // Bull. London Math. Soc. 1977. V. 9. P. 209-211.

[6] Lopez M. B. A negative result in differentiation theory // Studia Math. 1982. V. 72. P. 173-182.

[7] Stokolos A. M. An inequality for equimeasurable rearrangements and its application in the theory of differentiation of integrals // Anal. Math. 1983. V. 9. P. 133-146.

[8] Спринжук В. Г. Метрическая теория диофантовых приближений. М.: Наука, 1977.

Одесский государственный университет 